number of cases, in the absence of eyes in the young I have had several animals born with only one eye or one good eye, and the other sightless or imperfectly formed. Many are born with spots on the outer coating of the eye, which clear up under proper feeding. In animals on the scorbutic diet, eye trouble follows

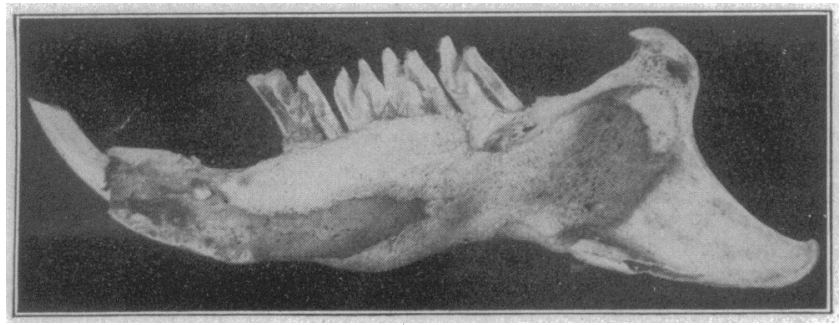

Fig. 7.-Thirty-three weeks: normal feeding about half of the time, with new bone formation.

even to the point of pus welling out over the eye during eating. Feeding orange juice is followed by complete clearing up of the trouble.

We have been working on monkeys for only about seven months, but arthritic disturbances are very pronounced; in two out of the four animals a condition resembling arthritis deformans occurs. The kneejoints enlarge to twice the normal size. The spinal column is curved, and the animal remains curled up, holding the head down. The mouth becomes filthy,

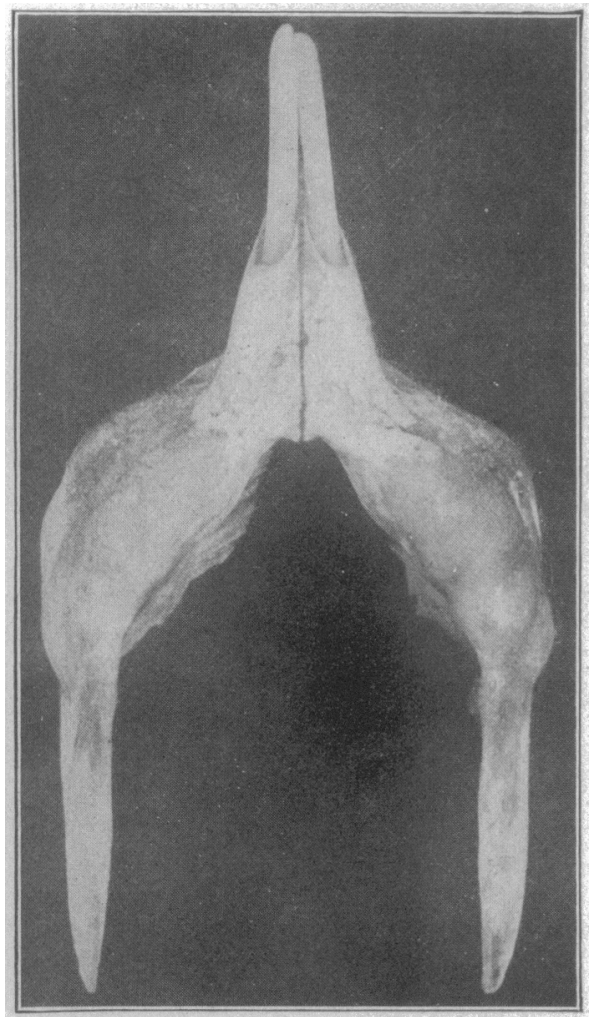

Fig. 8.-Fifty-sixth week: new bone formation complete; same series as in Figure 7 .

with excessive tartar formation, and the teeth become loose.

Exophthalmos occurred in the four experimental animals; large hemorrhagic areas have appeared in the supra-orbital region and under the eye. The eyes were covered with a film in one severe case. Blood trickled from the eye and mouth during an attempt to eat. The animal became temporarily blind. In all cases when orange juice was fed, the entire condition cleared up. If, however, the lens has become affected, it fails to clear, with the production of cataract. Cataract was so produced in one monkey and also in guinea-pigs.

The conditions described appear to be distinct from rickets. Unquestionably a proper supply of calcium and phosphorus is important, but, of the two, calcium seems to be the inorganic constituent that in modern diets is most likely to be deficient. Statistics and research indicate that the requirement, particularly for children, is greater than was formerly believed. It is well understood that calcium serves to regulate the equilibrium in almost any irregularity of the body mineral elements. But with all the proper food factors supplied except the antiscorbutic, the animal organism appears still to withdraw calcium from the teeth and some parts of the bone.

140 The Fenway.

\section{THE EFFECT OF DEFECTIVE DIETS ON TEETH}

\author{
THE RELATION OF CALCIUM PHOSPHORUS AND \\ ORGANIC FACTORS TO CARIES-LIKE AND \\ ATTACHING-TISSUE DEFECTS * \\ CLARENCE J. GRIEVES, D.D.S. \\ BALTIMORE
}

From its inception, dental literature has contained many statements associating food habit with oral disease. Dietary errors or excesses are said to be predisposing, if not active, causes of dental caries and gingival lesions. It is also implied that these diseases do not exist in races or individuals with correct food habits, the inference being that a proper dietary regimen will prevent oral pathologic conditions. Tangible evidence of what constitutes a correct diet is not offered by the donors of these hypotheses, which, when applied in daily routine, are not practical in the individual, whatever they may be en masse.

We are told that races living under primitive dietary conditions are free from oral disease: the Eskimo is said to be immune for the reason that he is essentially carnivorous, using little carbohydrate food; and the Maori, because he consumes quantities of carbohydrate and little protein food. In the midst of this exigency stands the fact that Anglo-Saxon races of all climes, with the greatest abundance and diversity of food, are being involved by oral disease to an alarming extent.

Function, or the lack of it, is said to be the predetermining factor in the induction of these diseases. The refinement of foods, which are softened by cooking, is cited to explain the prevalence of dental disease with every advance in civilization, in spite of the fact that persons of civilized countries obtain a larger percentage of better food per capita than those of primitive races. It is asserted, for example, that just as the consumption of refined cane sugar and bolted flour increases, so dental caries increases, and

* Read before the Section on Stomatology at the Seventy-Third Annual Session of the American Medical Association, St. Louis, May, 1922. 
today it involves 25 per cent. of the teeth of 85 per cent. of American schoolchildren.

For the most part, these statements are generalizations with as much foundation in fact as casual observation permits, though they all may contain elements of truth. There are so many qualifying and disturbing factors entering into any investigation of diet in relation to disease that the student is soon lost in a maze as to the relation of the simplest basal dietary elements to digestive and metabolic processes, not to mention the modifying influence the endocrine glands and pathogenesis may have on the resultant equation.

It is well, therefore, to hesitate before drawing conclusions as to the simplest diet fed an experimental animal, for it does not necessarily follow that these elements arrive in the cells of osseous and dental tissues without profound modification, which digestive and metabolic processes are bound to induce. This is well illustrated by the present tendency to associate deficiency diseases with absence of vitamins, neglecting more important basal dietary factors.

It is recognized that too great a simplification of the diet may, in itself, lead to deficiency disease; yet under these conditions the subject can be intelligently approached only by a study of diets which make for well-being in the animal and are as elemental as possible. That such dietetic study is feasible has been proved by E. V. McCollum and his associates in some 8,000 animal dietary experiments. For the sake of clarity in this report, but three basal dietary factors are considered: calcium, phosphorus and the organic substances existing in butter fat and cod liver oil; and these are considered in their relative concentration and percentage.

\section{ORAL CONDITIONS IN RATS}

It was my good fortune to study oral conditions in the same group of rats which were used in the recent research on rickets and related osseous pathology by McCollum, Simmonds, Shipley and Park, of the chemical hygiene and pediatric departments, Johns Hopkins University. Reports of these researches, which are too voluminous for quotation, are available to those interested.

Osseous and dental tissues are so closely related that the conclusions in these papers are here summarized:

These reports have shown that the internal structure of the skeleton of the rat could be changed at will by varying the ration which the animals received. In other words, bone is an extremely labile tissue and is readily influenced by nutritional environment. ${ }^{1}$

1. McCollum, E. V.; Simmonds, Nina; Shipley, P. G., and Park E. A.: Studies on Experimental Rickets. I, The Production of Rachiti

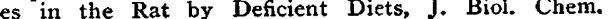
45: 333 (Jan.) 1921 .
A study of the effects of these diets on the skeleton would indicate that the growth of the skeleton was dependent on at least three substances: (1) an organic substance present in certain fats which may, or may not, be identical with the antixerophthalmic fat-soluble A; (2) calcium, and (3) phosphorus. ${ }^{2}$

If the organic factor is low in, or missing from, a diet, the structure of the osseous tissue is dependent on the ratio between the above mentioned ions in the diet and circulating blood.

If calcium is present in amounts equal to, or exceeding, those which would be optimal for growth and function and if all other factors are satisfactory but the phosphate ion is low, rickets is produced. ${ }^{3}$

The same disease results when the converse relation exists between the two ions."

Diets which contain optimal or excessive amounts of calcium but are low in phosphorus and the organic factor produce rickets.

Rickets also results from feeding diets low in calcium and the organic factor if phosphorus is present in satisfactory amounts.

Diets which are satisfactory except that they are deficient in the organic substance result in osteoporotic but perfectly calcified bone.

Diets which have a comparative deficiency in calcium but are very high in phosphorus and in the organic substance produce so-called osteosclerosis, with large numbers of small, imperfectly calcified trabeculae.

Diets deficient in calcium alone produce a pseudorachitic condition with overproduction of osteoid.

The studies of diets which contain varying percentages of calcium and calcium phosphate show that the absolute amount of either ion in the diet is of relatively little importance as compared to the ratio which exists between the two. That is, normally calcified bone is produced without regard to the reduction or diminution of either calcium or phosphate in the food, provided the content of the other ions is proportionately augmented or depressed.

\section{RESULTS OF DEFECTIVE DIETS}

Good growth, well-being, fertility and normal osseous tissues were induced in the rat when the relation of the total calcium was 0.64 and phosphorus 0.41 to $100 \mathrm{gm}$. by weight, with butter fat at 2.5 per cent. and cod liver oil at 1.5 per cent. of total diet, representing the organic factors, provided other dietary elements

2. Shipley, P. G.; Park, E. A.; McCollum, E. V., and Simmonds, Nina: Studies on Experimental Rickets, III, A Pathological Condition Bearing Fundamental Resemblances to Rickets of the Human Bein Resulting from Diets Low in Phosphorus and Fat-Soluble A: The Phosphate Ion in Its Prevention, Bull. Johns Hopkins Hosp. 32: 160 (May) 1921.

3. McCollum, E. V.; Simmonds, Nina; Shipley, P. G., and Park, E. A.: Experimental Rickets, VI, The Production of Rickets by Diets Low in Phosphorus and Fat-Soluble A, J. Biol. Chem. 47:507 (Aug.) 1921.

4. Shipley, P. G.; Park, E. A.; McCollum, E. V., and Simmonds, Nina: Studies on Experimental Rickets, XIX, Is There More Than One Kind of Rickets? Am. J. Dis. Child. 23: 91 (Feb.) 1922

5. Shipley, P. G.; Park, E. A.; McCollum, E. V., and Simmonds, Nina: Studies on Experimental Rickets, VII, The Relative Effectiveness of Cod Liver Oil as Contrasted with Butter Fat for Protecting the Body Against Insufficient Calcium in the Presence of a Normal Phosphorus Supply, Am. J. Hyg. 1:512 (July) 1921. 
were correct. In this paper, ${ }^{6}$ five diets are presented, which were selected from the fifty-seven diets previously reported when oral conditions were studied in relation to osseous disease.

That the dietary equation may be further simplified, the percentage of phosphorus in all of these diets is optimal $(0.41$ to the $100 \mathrm{gm}$.), while calcium and the organic factors in butter fat and cod liver oil are intentionally varied above or below normal percentages. This was accomplished "without making the deficiencies or concentration any greater than those which occur in the table of the modern American family." 6

It is emphasized that no sucrose exists in these diets; in one, 10 per cent. of whole milk powder occurs, which contains its small percentage of lactose. If oral fermentation has any part in inducing osseous lesions, the starches in which these diets abound may be active factors. These diets all contained ample water-soluble $\mathrm{B}$; as the rat synthesizes water-soluble $C$, the gingival lesions to be reported are not scorbutic, such as occur in the guinea-pig.

All diets were so finely ground as to prevent selection of food, and all experimental animals were adults and were placed on the diets at the age of about 60 days. They were taken, without selection, from the stock colony which lives on a stock diet under the best possible hygienic laboratory conditions and is free from other disease. It may be stated, as a control, that the stock rats in this colony show no caries-like or attaching-tissue defects

Eighty-five rats, about equally divided as to sex, were placed on the following diets:

Group 1, low calcium diets; Group 2, low calcium and low fat-soluble A diets; Group 3, high calcium and high butter fat diets (Figs. 1, 2 and 3).

It is most important, in considering the effect of diet on teeth, that the animal be an omnivorous feeder and that the teeth and attachments be comparable, numerically and histologically, to those of man. Guinea-pigs and rabbits, which ingest herbivorous diets of succulent roots and leaves, and in which the entire denture grows persistently, are not comparable in digestive function or omnivorous dietary to man.

Dogs, pigs and monkeys present ideal oral conditions for experimental study, but they litter infrequently and are prone to infectious diseases. They develop osseous defects, if confined under laboratory conditions

6. McCollum, E. V.; Simmonds, Nina; Kinney, E. M., and Grieves, C. J.: The Relation of Nutrition to Tooth Development and Tooth Preservation, Bull. Johns Hopkins Hosp. 33:376 (June) 1922. and, if allowed freedom, obtain small but definite food factors which might modify the experiment.

The disadvantages of the rat denture, which is small and presents histologic difficulties, are more than offset by assurance of correct experimental conditions. Rats are omnivorous and are prolific breeders, so that many generations can be studied. They do not suffer from confinement or monotonous laboratory diet.

In the denture of the rat, the four incisors grow from persistent pulps, while the twelve molars are coronally and apically completed teeth, all of which are erupted by the fortieth day. The cusps are of the bunodont type, associated with omnivorous diet rather than the lophodont type, associated with the herbivorous diet, as in the guinea-pig.

While the rat's incisors are subject to resorption and repair by persistent pulp function, like incisors and molars of guinea-pigs, the rat's molars are coronally and apically finished teeth and, except for size, numerically and histologically comparable to the human molars. Excellent enamel surfaces in perfect proximate contact; simple dentin of the ortho variety and large pulps with evident high resistance are all excellent indicators of any lesion.

Subgingival attachments are dense and wonderfully adapted to function; the roots present definite cemental laminae with closed and pointed apexes and multiple foramina. Well-defined periodontal membranes are attached by stout alveoli, furnishing excellent support to gingival crests which lie in perfect contact and thus protect embrasures.

In the rat, the relation of masticatory function to diet is ideal; like all rodents, the rats must literally "chew for dear life"; whether the diet be soft or hard, the denture is used until disintegrated, and all teeth are abraded by their own interaction. Any disturbance of this highly vital nutritional function in adult rodents must, indeed, be fundamental. Under such conditions, it is therefore interesting to find caries-like and attaching-tissue lesions involving teeth of adult rats when placed on the foregoing diets, with no such defects in the stock controls.

Percentages of lesions are calculated on the number of teeth involved (twelve molars); the number of rats placed on these diets, and the period on the diet, which averaged 120 days.

Thirty-six per cent. of the males and 44 per cent. of the females showed caries-like and attaching-tissue defects, with increasing percentages in the littering rat, the oral condition of which is most interesting.

In Group 1, the low calcium diets (Fig. 1), phosphorus and fat-soluble A were nearly optimal, while 
calcium was about one-half the normal: the diets are thus simplified to one defect, with all other factors well balanced. When the ash of a diet is acid, as in some of these, it appears to increase the tendency to lesions. Twenty-two per cent. of the rats on these diets showed caries-like defects, and 3.65 per cent. of their molars were involved. Forty-one per cent. displayed attaching-tissue lesions which involved the molars to the extent of 57.9 per cent.

It has been noted that "diets deficient in calcium alone produce a pseudorachitic condition with overproduction of osteoid." Skeletal defects were pronounced in this group, and a large percentage of alveolar involvement was anticipated. It cannot be assumed, however, that diets which affect long bones will always affect tooth attachments, for such is not the case.

It is also evident from the contrasting percentages of caries-like lesions, 3.65 per cent., and attachingtissue defects, 57.9 per cent., that, as far as dietary factors are concerned in the induction of these lesions, they are not similar in either incidence or type.

In Group 2 (Fig. 2), the low calcium and low fat-soluble A diets, phosphorus was optimal, while calcium was one third of the normal, and fat-soluble $\mathrm{A}$ was relatively low; this produced two deficiencies, with all other dietary factors well balanced. Thirty-one per cent. of the rats on these diets showed carieslike defects, and 5.21 per cent. of their molars were involved. Twenty per cent. $d$ is $p 1$ a y e attaching-tissue defects which involved the molars to the extent of 17.7 per cent.

The contrast in this group is remarkable. A twofold deficiency, which in the previous study of fiftyseven diets produced the greatest percentage of caries, 5.21 per cent., in this type of diet produced only 17.7 per cent. of attaching-tissue lesions, which was next to the lowest percentage in the previous series of 220 rats.

It has been noted that "rickets results from diets low in calcium and the organic factor, if phosphorus be present in satisfactory amounts." The fact that these diets, which produced experimental rickets and a large percentage of caries-like lesions in rats, induce such comparatively slight attaching-tissue disturbance cannot for the moment be explained. It appears to confirm the clinical observation that, when human caries involves the adult denture, the incidence of gingival disease is low.

It must not be concluded that diets which produce experimental rickets also induce caries-like lesions. A large group of animals in the previous report were definitely rachitic, yet, macroscopically, suffered few oral defects of any type. This low calcium and low fat-soluble A diet is here reported for the very reason that rachitic and caries-like lesions are rarely coexistent.

As there is a prejudice against comparing deductions from animal experimentation with human experience, it might be well to explain these percentages.

With the rat's life cycle at 1 to 30 for the human, a rat from 120 to 150 days old is comparable to a child between the tenth and thirteenth years, especially when differences in developmental cycles are considered. At these ages, the child's teeth are said to be 25 per cent. carious; but twelve, or 50 per cent., less teeth are exposed to carious processes in rats, when compared with an average of twenty-four teeth in children, and 12.5 per cent. represents a like reduction of the 25 per cent. of child caries. When the 12.5 per cent. for the child is compared with 5.21 per cent. for the rat, and when the period of exposure, which was much shorter in the rat, is considered, the discrepancy is not so great as might appear.

The incidence of human gingival disease in adults cannot be stated; it is evidently high, but that it so rapidly involves persons, or their teeth, in any such percentages as here presented for the rat is questionable.

In Group 3 (Fig. $3)$, the high calcium and high butter-fat diets, phosphorus and fat-soluble A were optimal, but calcium was twice the optimum. This produced but one deficiency, with all other dietary factors in balance. Seventeen per cent. of the rats on this diet showed caries - like defects, and 1.73 per cent. of their molars were involved. Twenty-eight per cent. displayed attaching-tissue defects which disturbed 33 per cent. of the molar attachments.

It has been explained that high calcium diets produce good growth but early deterioration, with pseudorachitic changes in bone, particularly in the second and third generations.

The lesions resulting from this diet, 1.73 per cent. caries and 33.3 per cent. attaching-tissue lesions, are in direct contrast to the low calcium and low fatsoluble A diet, in which the caries-like defects were the highest, 5.21 per cent., and the attachment disturbance nearly the lowest, 17.7 per cent., in the entire series.

\section{CARIES-LIKE LESIONS}

The essentials of dental caries, according to the modern conception, are environmental. It is believed that caries progressively invades teeth from without inward and that it may result from chemicomicroorganic causes in any retention center. Whatever the bacterial types, they are presupposed to produce sufficient acidity to decalcify enamel and dentin, and to be sufficiently proteolytic to complete dentinal destruction. 
In this conception it is not denied that teeth may not be predisposed to caries from developmental structural defects, which may resuit in early formative periods and may assist in localizing carious lesions. The finest types of enamel and dentin may be invaded in the same mouth, and even the same tooth, while hypoplastic enamel and dentin may escape.

Other than this distinction, it does not admit that completed teeth are weakened from within by resorption of dentin, or interglobular space formation arising from dental pulp function; or that enamel may be softened from within, or that it is permeable or vital.

These facts are reviewed for the reason that there have appeared certain sections of rat's molars which suggest some internal factor in human dental caries, but they do not occur in these groups of diets.

The lesions in these groups always proceed from without inward and do not result from hypoplastic enamel, nor are they absorptions from dental pulp function. Clinically, they are identical with human caries.

The minute and slowly enlarging initial lesion in any deep enamel sulcus; the rapid dentinal invasion, undermining enamel walls; the typical laminated carious dentinal detritus, and the final pulp exposure, followed by subapical bone involvement and alveolar abscess, are classic, macroscopically.

The distal sulcus of the first molars and the central sulcus of the second molars are usually seats of initial lesions, which may be due to food retention. Mesial and distal contact facets are occasionally secondarily involved, but they are not primarily attacked as in man. This immunity may arise from the excellent proximate contact and the protection of embrasures by attaching and gingival tissues.

These lesions were stud-

ied in ground and decalcified sections. Coriell's lapping machine was used in grinding, and Mueller's fluid was used in decalcification. By the latter method, occasional slides were obtained without complete loss of enamel. Slides were stained with thionin, silver and carmin.

Microscopic sections show slow decalcification of enamel, either by loss of inter-rod substance or complete disintegration of both rods and cement substance. Dentinal invasion spreads typically, undermining enamel along the dento-enamel junction. Tubules are enlarged and invaded irregularly; characteristic areas appear, showing complete loss of matrix and projection of fibrils into masses of proteolyzed dentin containing bacteria. Dentinal destruction seems much slower and more regular than in human caries. Frequently observed tubular calcification and osteodentin formation indicates a pulpal resistance much higher in the rat than in man. Pulp involvement and exposure is not unusual. In old cases, the entire tooth becomes dark and necrotic and apical abscess results, with typical apical denudation and destruction of subapical tissues. In some instances, granulomatous or cystic types of apical disease occur, presenting symmetrical bone areas well walled off by condensing osteitis and lined by fibrous tissue, as the stains indicate. In others, much bone liquefaction results, with fistulas. These are evidently of the purulent type, and occasionally result in large osteomyelitic areas.

\section{THE ATTACHING-TISSUE LESIONS}

In reporting these defects, "only such gross osseous lesions as actually exposed cervical aspects of roots or finally caused loss of teeth are presented." The flare of the rat's molar roots is so great and they are so well attached that it may be assumed that such teeth are not lost without the persistence for some time of soft tissue lesions. "Attaching-tissue defects occur in two groups: $(a)$ the atrophic types, with progressive horizontal and regular loss of alveolar crests, the cortex remaining hard, rounded and smooth, and $(b)$ the infective type, with irregular vertical invasion of alveolar walls and localized root exposure, in which the cortex is rough, porous or missing," as in true pyorrhea. "In short, all phases of the osseous lesions of gingival disease occur as in the human." 6 In both maxilla and mandible, the lingual crests are frequently lost. The disease may localize about one molar or may be general. If complicated by caries and loss of proximate contact, food pack and trauma soon destroy the tooth.

Microscopic slides show much involvement of al-
osteoporotic or cartilaginous, but which still remain intact, supporting the gingival crests.

Occasional peninsulas of bone persist, but trabeculae are indefinite and canalization frequently results. There is much osteoid and round cell infiltration. Deep pockets occur, lined by epithelium, supported by the remaining periodontal fibers, but it cannot be said that they arise by central invasion of the periodontium along lymphatic vessels, as in true gingival disease. Cervical deposits of calculi were noted in some instances: its character together with that of the soft tissue lesions will be reported later.

While only certain rats suffered from caries-like and attaching-tissue lesions, as the percentages indicate, the impression must not obtain that the two lesions did not appear simultaneously in the same rat, for fully one third of the group were so involved. 
These experiments had none of the mathematical accuracy of the feeding to guinea-pigs of scorbutic diets, which are said to produce 100 per cent. scurvy.

The varying percentages of damage to tissue present a remarkable resemblance to human disease; they indicate the resistance of the individual rat to these diseases. Its immunity, its superior digestive and metabolic processes, or possibly other factors, evidently protected its teeth and attachments against invasion.

The constancy of the character of caries-like lesions is interesting. From what has been observed, no matter what the dietary defects, they are remarkably alike in microscopic section. Quite the reverse is true of the diseased attachments, the pathologic character of which differs materially, but it is not possible as yet to state the type of lesions induced by any particular diet. Indeed, the entire phenomena run so true to clinical experience as to tempt one to conclusions which might be premature, for, as in man, these lesions appear to result from all types of dietary defects, and it would appear that the animal economy can form and maintain a fine denture, with attachments, on very restricted diets, provided the animal be in proper dietary balance.

In the absence of sucrose, and with starch as the only oral fermentative medium, it is possible to indulge in many fascinating and plausible hypotheses as to the cause of dental caries, all of which are likely to be wrong. It might be reasoned, since the largest caries-like percentages occurred in Group 2, which contained 10 per cent. whole milk powder, that even this percentage of lactose might have increased the fermentation in the starches in the diet and in-

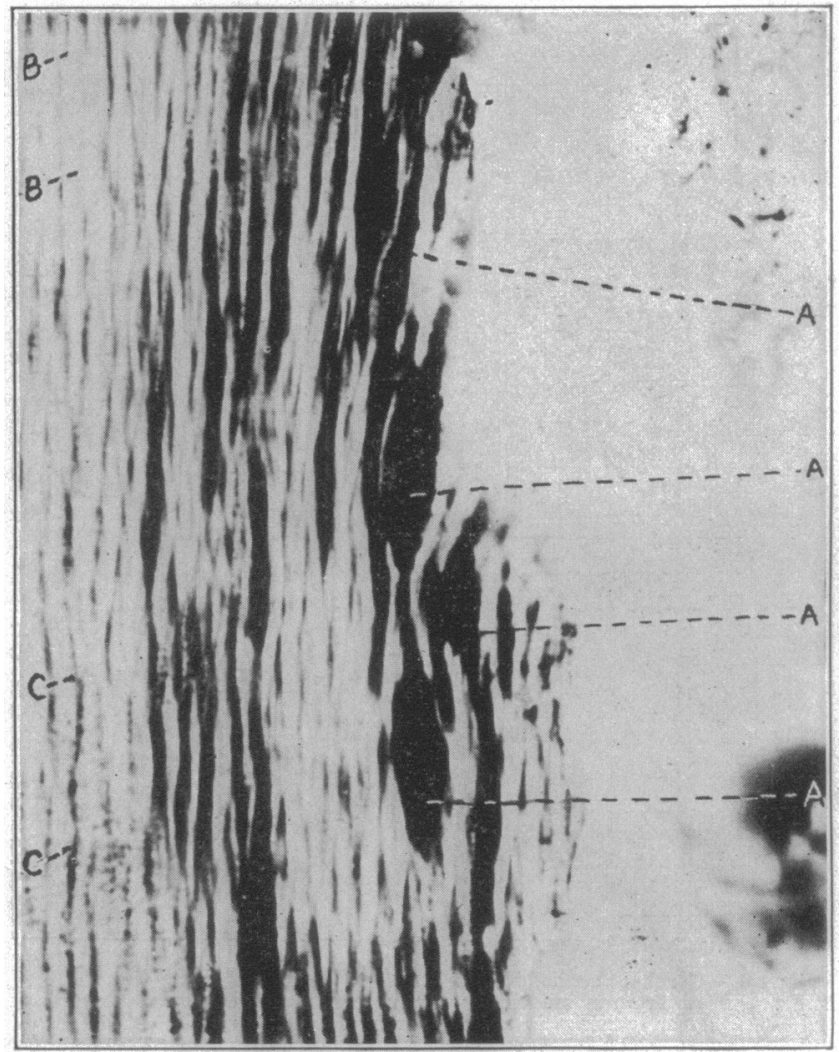

Fig. 5.-Field from Figure 4, reduced from photomicrograph, $\times 800$ $a$ carious tubules, liquefaction foci; $b$, dentin slightly involved; $c$, deeper invasion. duced dental caries, were it not for the fact that the stock diet contains a larger percentage of milk and the teeth of stock rats are not carious. From certain evidence, it might be said that disturbances of the calciumphosphorus balance of the blood, of tetany and rickets, were the main factors in caries; but nearly all children have carious teeth and few children are rachitic or involved by tetany. The reverse is equally true, while very few children, as compared with adults, suffer from gingival disease.

The intricate relation of calcium and phosphorus in the blood to the endocrine secretions, and to tetany and rickets, has been explained by Howland, Tisdall and Kramer. ${ }^{7}$

7. Howland, J., and Kramer, B.: Calciun Serum in Relation to Rickets, Am. J. Dis. Child. 22: 105 (Aug.) 1921. Kramer, B.; Tisdall, F. F., and Howland, J.: Observations on Infantile Tetany, ibid. 22: 431 (Nov.) 1921.
"Tetany is an expression on the part of the nervous tissues of an insufficiency of the calcium ion; rickets is an expression on the part of the skeleton of disturbed relations between the calcium and phosphate ions. The reason that tetany is so frequently associated with rickets is that rickets is a disease in which the calcium ion in the body tissues and fluids is subject to wide variations." 4 Both of these diseases depend on the calcium-phosphorus balance in the blood, but may occur independently of each other.

Typical rachitic lesions and symptoms are known to result from two types of disturbance as indicated by the blood, i. e., "one in which calcium was normal and phosphorus low (low phosphorus rickets) and the other in which phosphorus was normal and calcium low (low calcium rickets) and there may be others."

Alveolar lesions, which are exposed to oral infection and occlusal trauma, are hardly likely to be more concrete than the internal lesions of rickets. On the contrary, they might be expected to present many more complicated pathologic expressions of simple dietary defects.

This hypothesis cannot as yet be applied to dental caries. It is reasonable to suppose that disturbance of the osseous attachments of teeth might occur from within outward, by the vascular pathway, and that gingival disease might result from the same internal etiologic causes as rickets; but dental caries is an invasion of the tooth, arising externally, and proceeding from without inward. Therefore, one must think of the oral environment as permitting such invasion.

In these specimens, caries-like lesions are so far from being an internal disturbance, or resorption of enamel and dentin by pulp function, that the pulp actually protects its vascularity by tubular calcification and secondary dentin formation.

Malfunction of the endocrine glands has been enthusiastically advocated as the etiologic factor in dental caries; these assertions are based on clinical observations of hypoplastic and dystrophic teeth in cretins and in patients with acromegaly. Similar lesions are said to result from syphilis, exanthems and artificial infant foods. Certain defects in the teeth of dogs, of monkeys and of rats are reported to result experimentally from damage to the endocrine glands, particularly the thyroid and parathyroids.

In a previous paper, ${ }^{6}$ it was shown that many of these defects could be duplicated in rat's teeth by the use of defective and deficient diets, with no apparent symptoms of endocrine disturbances. Unless the 
animal's diet is known and definitely planned, according to modern dietary usage, these conclusions as to the relation of endocrine experimental malfunction and oral disease are useless and misleading.

I am aware of the recent work of McCarrison ${ }^{8}$ and Broderick. $^{9}$ It seems highly probable that defective endocrine function and disturbed metabolism may be intimately associated with the use of defective and deficient diets, and that any, or all, of these factors may play an etiologic rôle in oral disease; but these correlations are as yet hypothetic.

Until further facts are available, one can think only of the necessity for a proper calcium-phosphorusorganic factor balance in any diet as most important in the formation and maintenance of normal bones and teeth and healthy attaching-tissues.

201 West Madison Street.

\section{ABSTRACT OF DISCUSSION}

OF PAPERS OF DRS. HOWE, GRIEVES AND SHIPLEY

Dr. Eugene S. Talbot, Chicago: These papers confirm some research work I did thirty-five years ago. The work of Dr. Howe illustrates better than anything that has ever been published the bone absorption that is due to change in diet. My researches were done on animals, such as cows, hogs, horses, etc. Any animal that is changed from its normal environment and food will have a certain amount of absorption of bone and this absorption is not due to infection or inflammation. Persons who are apparently in good health have absorption of bone tissue at some time or another. The only way we can demonstrate that fact is by an examination of the alveolar process. The alveolar process is a transitory structure. It is not a stable structure like other bones of the body. When the proper food is not taken into the system the alveolar process will begin to absorb. About twentyfive years ago I examined the mouths of men working in mountains in Switzerland, building railroads, and found that the men working in the shafts and tunnels, in dark places, without sunshine and proper air and with poor food, had absorption of the alveolar process. In many cases the teeth became so sore they had to be extracted. I examined thousands of men returning from the Philippines and Cuba and found the same thing. Many similar cases are reported occurring among English soldiers in South Africa at the time of the Boer War. They called it scurvy, but there were no constitutional symptoms. The teeth became sore and loosened. just what kind of food should be given to one person and what kind to another under similar condition is a question. We do not know what foods will preserve the alveolar process in different persons.

Dr. Percy R. Howe, Boston: Scientists are beginning to get away from the present day theories of dental caries. As Dr. Shipley has said, we know almost nothing about it, but a few facts were brought out that show the importance of studying the problems from a dietiary point of view.

8. McCarrison, R.: Studies in Deficiency Disease, Oxford Press, 1921 9. Broderick F. W.: The Effect of Endocrine Derangement on the Teeth, Brit. Det. J. 41: 955 (Oct. 15) 1920.
Dr. Paul G. Shipley, Baltimore: The American diet at the present time is perilously near the danger mark. If you go down to the "quick lunch" at noontime, and watch the tired business man lunch you will see him eat a ham sandwich, and a piece of pie of doubtful origin and drink a cup of coffee. His breakfast in the morning consisted of cereal ready done, alleged cream or pasteurized milk, sugar, more coffee, and toast if he is fastidious and has a lot of time. His dinner consists of muscle meat, steak, chops, or something of that sort (only occasionally a bit of liver), pie or some sort of sweet. With these he has some well cooked vegetable and potatoes, sometimes a salad. Whatever vitamins may be contained in this food, he is hovering close to a very pronounced deficiency in them all. Not only is this the case, but he is taking a diet which is probably extraordinarily bad in the way of its salt content. His steak contains almost no calcium, but is high in phosphorus. The salts of milk form quite a perfect mixture, but nobody seems to know just what happens to milk after the dairyman gets through with it. If you ever have the opportunity to inspect the tanks in which milk is pasteurized, or the cylinders on which milk is dried, you will quickly become convinced that a pronounced change has taken place in the salt content of the milk. Where milk is dried, it is necessary every day to take down the apparatus and chisel off from the sides of it a scale which is nothing more or less than the calcium phosphate which is thrown out of the milk during the process of drying. You are all familiar with the skin which appears on the top of milk when it is brought to the boiling point. More of this material sticks to the sides of the utensil in which boiling is done. Besides protein and some fat, that skin contains a large amount of calcium phosphate, which is lost to the consumer. Dietetics is today a little known field and it seems to me that the best we can do at present in prescribing a regimen is a general, varied diet with, as Dr. Howe has suggested, some fresh, uncooked food every day. Dr. Howe spoke of magnesium and its increase in certain pathologic conditions. By substituting magnesium for calcium in the diet of animals we are able to produce lesions which are very closely related to and, under certain conditions, identical with the rickets seen in children. That
it is not altogether unimpor tant as you will understand when you remember that cow's milk contains a much greater quantity of magnesium than does the breast milk of the mother with which nature intended her child should be fed.

Relative Importance of Fatal Accidents.-The importance of fatal accidents among the industrial policy holders of the Metropolitan Life Insurance Company is brought out strikingly by a comparison with certain diseases of primary interest to public health. There were only twenty-nine death claims for typhoid fever, for example, paid in New York in 1921 , as compared with 1,022 death claims paid for accidental deaths. During that year, twenty-eight claims were paid on account of measles, ninety-six on account of scarlet fever, thirty-four on account of whooping cough, and 213 on account of diphtheria. Thus we see there was over two and a half times as much mortality from accidents in the city as from all four of these epidemic diseases of childhood.-Statistical Bulletin, September, 1922. 